Das jüdische Jugendbuch 
Gabriele von Glasenapp und Michael Nagel

\section{Das jüdische Jugendbuch}

Von der Aufklärung bis zum Dritten Reich

VERLAG J. B. METZLER

STUTTGART · WEIMAR 
Die Deutsche Bibliothek - CIP Einheitsaufnahme

\author{
Glasenapp, Gabriele von: \\ Das jüdische Jugendbuch : \\ von der Aufklärung bis zum Dritten Reich / \\ Gabriele von Glasenapp und Michael Nagel. \\ - Stuttgart ; Weimar : Metzler 1996 \\ NE: Nagel, Michael:
}

ISBN 978-3-476-01413-9

ISBN 978-3-476-03638-4 (eBook)

DOI $10.1007 / 978-3-476-03638-4$

Dieses Werk einschließlich aller seiner Teile ist urheberrechtlich geschützt. Jede Verwertung außerhalb der engen Grenzen des Urheberrechtsgesetzes ist ohne Zustimmung des Verlages unzulässig und strafbar. Das gilt insbesondere für Vervielfältigungen, Übersetzungen, Mikroverfilmungen und die Einspeicherung und Verarbeitung in elektronischen Systemen.

(C) 1996 Springer-Verlag GmbH Deutschland

Ursprünglich erschienen bei J.B. Metzlersche Verlagsbuchhandlung und Carl Ernst Poeschel Verlag GmbH in Stuttgart 1996 


\section{EINLEITUNG}

Diese Studie versteht sich als ein Beitrag der Kinder- und Jugendbuchforschung zur Geistesgeschichte der Juden im deutschsprachigen Raum von der Aufklärung bis zum Dritten Reich. Sie möchte an eine dringende, dabei jedoch letztlich keiner Lösung zugeführten grundsätzliche Fragestellung erinnern, mit der sich das Judentum als konfessionelle Minderheit innerhalb einer zuweilen toleranten, häufig aber abweisenden oder gar feindseligen Umgebung für diesen gesamten Zeitraum immer wieder von neuem konfrontiert sah: Welches Erziehungs- und Bildungsziel, welche Hilfen zu einer Identitätsfindung innerhalb der nichtjüdisch geprägten Gesellschaft konnten und wollten jüdische Pädagogen, Autoren und Rabbiner den ihnen anbefohlenen jüdischen Kindern und Jugendlichen vermitteln? Die verschiedenen Positionen zu diesem Anliegen, der jüdischen Jugend einen Weg und eine Bestimmung nahezubringen, werden hier am Beispiel der zeitgenössischen jüdischen Jugendbuchkritik und -programmatik dargestellt.

Die Forschung zum historischen Kinder- und Jugendbuch untersucht neben der betreffenden Primärliteratur, also vor allem den an den Adressatenkreis von Kindern und Jugendlichen gerichteten Werken mit ihrem Entstehungszusammenhang, ihrer Rezeptionsgeschichte und weiteren Aspekten auch die begleitende Lektürepädagogik, also die - im weitesten Sinne - mit Lektüre verbundene erzieherische Intention. Diese äußert sich, sieht man einmal von einer den Primärtexten stets zueignenden immanenten Zielrichtung ab, im Einzelfall zunächst in den Konzepten, Kontroversen und Kritiken zu der speziell für die Heranwachsenden bestimmten Literatur. In einem weitergefassten Überblick lassen sich, ausgehend von einem Ensemble entsprechender Quellentexte, bestimmte Einstellungen, Hoffnungen und Absichten in Beziehung zu den geistesgeschichtlichen Strömungen ihrer Zeit setzen. Dabei tritt häufig - bei gleichzeitiger Bindung an die Tradition und ausgehend von der Gegenwart - eine betont der Zukunft zugewandte Sichtweise hervor: Es geht den Autoren, Lehrern und Geistlichen, welche Beiträge zur Gestaltung der Kinder- und Jugendlektüre sowie entsprechende Vorworte und Rezensionen verfassen, in der Regel um eine - etwa religiöse, ideelle, lebenspraktische oder wie auch immer geartete Einstellung, die sie der besonders empfänglichen Jugend, als literarisches Modell dargeboten, für den späteren Lebensweg vermitteln wollen. Auch das Kinder- und Jugendbuch selber hat die Absicht, in bestimmter Richtung, dabei aber meist unausgesprochen auf seine Leser einzuwirken. In der Konzeption und Diskussion der Jugendliteratur, welche ihr Forum nicht selten in eher peripheren Publikationen wie Schulprogrammen, Vorträgen, Briefwechseln etc. findet, treten derlei Tendenzen je- 
doch offener, oft auch vollständiger, in Erscheinung. Sie können in ihrer Individualität, ihrer Benennung auch von emotionalen und mentalen Zusammenhängen mitunter ein deutlicheres Bild von Erziehungsinhalten und -zielen abgeben als dies die "offiziellen" pädagogischen Texte ihrer Zeit vermögen. Die Beschäftigung mit lektürepädagogischen Äußerungen hat der Kinder- und Jugendbuchforschung inzwischen eine Reihe von Beiträgen zu unterschiedlichen Fragestellungen oder Epochen, so etwa zur Aufklärung oder zur Jugendschriftenbewegung, eingebracht.

Hier wird nun erstmals ein derartiger in sich geschlossener Beitrag über einen längeren Zeitraum vorgelegt, der außerdem auf einen bisher kaum beachteten Adressatenkreis, nämlich jüdische Kinder- und Jugendliche, eingeht. Der Charakter einer Grundlagenforschung ergibt sich für die vorliegende Darstellung vor allem wegen des an zweiter Stelle genannten Sachverhaltes: Erst in der jüngsten Zeit ist das jüdische Kinder- und Jugendbuch selbst überhaupt zum Gegenstand wissenschaftlichen Interesses geworden. Auch in Fachkreisen war bisher die bloße Existenz von Werken, die, von jüdischen Autoren für die heranwachsenden Glaubensgenossen verfaßt, eine spezifisch jüdische Themenstellung besaßen, so gut wie unbekannt. Die vereinzelten Beiträge jüdischer Wissenschaftler zum jüdischen Schul- und Jugendbuch aus den zwanziger und dreißiger Jahren des zwanzigsten Jahrhunderts sind in Vergessenheit geraten. Damals begann sich ein Interesse jüdischer Pädagogen für die Geschichte des spezifisch jüdischen Kinder- und Jugendbuches abzuzeichnen, welches jedoch durch das Dritte Reich jäh abgebrochen und bis vor kurzem nicht wieder aufgenommen wurde. Demzufolge haben auch in den wenigen neueren Beiträgen zur Geschichte der jüdischen Erziehung, seien diese nun als Überblikke über einen bestimmten Zeitabschnitt oder als regional orientierte Untersuchungen abgefaßt, Fragen der literarischen Sozialisation bislang kaum eine Beachtung gefunden.

Das vorliegende Buch, gegliedert in zwei von jeweils einem Autor bearbeitete Hauptabschnitte, möchte in die Fragestellung nach den Zielen und Äußerungsformen jüdischer Lektürepädagogik zwischen 1774 und 1942 einführen. Dies geschieht sowohl mittels einer chronologisch-thematischen Übersicht als auch mit Hilfe von einzelnen detaillierter dargestellten Ausschnitten. Um mit einiger Sicherheit die bislang nicht einmal ansatzweise bekannten inhaltlichen Verlaufslinien der zeitgenössischen Auseinandersetzungen über geeignete oder ungeeignete Lesestoffe für die jüdische Jugend, um die Konzeption und Rezensionstätigkeit von spezifisch jüdischen Schulbüchern, religiösen Unterweisungen und erzählender Literatur nachzeichnen zu können, bedurfte es einer relativ breit angelegten Sichtung und Verarbeitung von aussagefähigen Dokumenten zum Themenbereich. Eine Reihe von Fragestellungen konnte bei dieser ersten, notwendig beschränkten Beschäftigung mit dem Untersuchungsgegenstand verständlicherweise nur angesprochen werden. Es versteht sich insbesonders, daß es hier nicht beabsichtigt war, zur Erziehungswirklichkeit jüdischer Kinder und Jugendlicher, insbesonders zu der Frage nach ihrer tatsächlichen Lektüre und nach deren Auswirkungen, repräsentative Aussagen zu gewinnen.

Zeigen sich nun grundsätzliche und bleibende lektürepädagogische Einstellungen beim Judentum von der Berliner Aufklärung bis zum Dritten Reich; läßt sich ein 
roter Faden, eine dominierende Intention für den hier untersuchten Zeitraum erkennen? Die Einstellungen zur jüdischen Kinder- und Jugendlektüre, im Wandel der Epochen betrachtet, machen durchgängig deutlich, daß seit Mendelssohns Tagen das Verhältnis zur nichtjüdischen Umgebung für die Überlegungen der jugendliterarisch engagierten Rabbiner und Pädagogen eine große, teils sogar eine bestimmende Rolle spielte. Die an der Diskussion beteiligten Personen mußten in der Gestaltung ihrer für die Jugend bestimmten Schul- und Erzählliteratur jedoch - vor allem in den ersten Jahrzehnten nach dem Beginn der Reformen - gleichzeitig Rücksicht auf eine jüdische Bevölkerung nehmen, die bis in die zweite Hälfte des neunzehnten Jahrhunderts hinein noch vielfach in der Tradition lebte und dachte. Zwischen diesen beiden Polen bewegen sich die Überlegungen zur Einrichtung einer geeigneten Jugendlektüre; sie spiegeln dabei die Unsicherheit und die Meinungsvielfalt wieder, die das Reformjudentum seit der unumgänglichen Aufgabe des voremanzipatorischen, universell-religiösen Erziehungskonzeptes bewegten. Welche jüdischen Charakteristika sollte etwa ein Lesebuch aufweisen, in welcher familiären oder gesellschaftlichen Umgebung konnte eine Erzählung für die jüdische Jugend spielen? Sollten die jüdischen Kinder- und Jugendschriften vor allem einen Beitrag zur Erziehung aus dem Ghetto heraus leisten, oder sollten sie die Heranwachsenden in einer Zeit nachlassender jüdischer Religiosität wieder zum Bewußtsein jüdischer Geschichtlichkeit und Tradition führen? Wie sollte man im Bereich der jugendlichen Lektüre die - wie Max Wiener sich 1933 ausdrückt - „bestimmte Dosierung des aufrecht zu erhaltenden Judentums«, vor allem also den zentralen Stellenwert des Religionsgesetzes, bemessen? Dergestalt beleuchtet der Diskussionsverlauf bis zur Mitte des neunzehnten Jahrhunderts die Problematik der Reformbestrebungen bei gleichzeitiger Bewahrung von Traditionen.

Eine festere Position zeigt hier erst die ab etwa 1860 verstärkt publizierende NeoOrthodoxie. Seit der Jahrhundertwende versuchen jüdische Pädagogen und Autoren dann, sich in bestimmtem Maße auch auf die Jugendschriftenbewegung zu beziehen, müssen sich jedoch mit deren Postulat der Tendenzfreiheit auseinandersetzen. Andere folgen bald darauf eher zionistischen Konzepten. Die erzwungene kulturelle Dissimilation seit 1933 beendet die ohnehin eingeschränkten Möglichkeiten zu einem eigenen jugendliterarischen Gestalten, indem nun für das jüdische Jugendbuch jegliche Aussage zu der - schon so lange selbstverständlich gewordenen - kulturellen und geschichtlichen Verbindung mit der nichtjüdischen Umgebung unmöglich geworden ist: Ein neues geistiges Ghetto ist von den Machthabern angeordnet worden und wird mittels einer akribischen Zensur überwacht. Mit der Resignation der meisten Juden am Assimilationsgedanken geht jedoch auch eine Rückbesinnung auf jüdische Besonderheiten und Traditionen einher, die den Kindern und Jugendlichen, etwa mittels Palästina-orientierter Erzählungen, Perspektiven aus der unmenschlich gewordenen Umgebung heraus zeigen will. Die 1943 verordnete Einstellung des letzten noch verbliebenen jüdischen Presseorgans markiert gleichzeitig das Ende der Beschäftigung mit jüdischer Kinder- und Jugendliteratur im deutschsprachigen Raum.

Zum Charakter des Untersuchungsmaterials: Als Quellentexte für die hier vorliegende Darstellung wurden neben Vorworten jüdischer Kinder- und Jugendbücher 
auch Schulberichte bzw. -programme, Ankündigungen privater Lehranstalten und Lehrpläne sowie Beiträge, Rezensionen und kommentierte Ankündigungen in jüdischen Zeitungen und Zeitschriften genutzt, ferner Briefwechsel, Vorträge, (ideelle) Testamente, Vorschlags- und Auswahllisten zur häuslichen Lektüre oder für Schulbibliotheken, daneben zum Teil auch Autobiographien, Biographien und allgemeinpädagogische Beiträge, wenn diese auf kindliche und jugendliche Lektüre Bezug nahmen. Für die Erhellung des Zusammenhanges lektürepädagogischer Ausrichtungen mit der Geistesgeschichte des Judentums im deutschsprachigen Raum wurde auf erziehungsgeschichtliche Beiträge, auf jüdische Literaturgeschichten sowie auf die neuere einschlägige historische Literatur zurückgegriffen, zum Vergleich mit Positionen der nichtjüdischen Lektürepädagogik und der allgemeinen Pädagogik ebenfalls auf die entsprechenden, zumeist neueren Beiträge. Orientierungshilfe bei biographischen Fragestellungen gaben neben den allgemeinen und speziellen Nachschlagewerken dankenswerterweise auch die Bestände des Archivs Bibliographia Judaica in Frankfurt a.M.. Zu Begriffsbestimmungen wurden vor allem drei Lexika herangezogen: Encyclopaedia Judaica. Das Judentum in Geschichte und Gegenwart [Dt.Ausg.]. Bd.1-10 [A-Lyra. Mehr nicht ersch.]. Berlin 1928 - 1934; Jüdisches Lexikon. Ein enzyklopädisches Handbuch des jüdischen Wissens. Begründet von Georg Herlitz und Bruno Kirschner. Bd.1-[5]. Berlin 1927 - 1930 sowie Encyclopaedia Judaica [Engl.Ausg.]. Bd.1-16. [Nebst] Yearbook 1973 f. - [u.] Decennial Book 1973/82. Hrsg. Cecil Roth. Jerusalem 1971 f. Die bibliographische Orientierung hinsichtlich der Quellentexte und auch deren Standortbestimmung wurde, vor allem zu Beginn der vorliegenden Untersuchung, wesentlich durch die Vorarbeiten und gleichzeitigen Recherchen eines Forschungsprojektes zur jüdischen Kinder-und Jugendliteratur (einer Kooperation des Instituts für Jugendbuchforschung, Univers. Frankfurt a.M. mit dem Porter-Institute der Univers. Tel Aviv sowie dem Institut für Popular- und Kinderkultur der Univers. Bremen) erleichtert, an welchem die Autoren des vorliegenden Buches ebenfalls mitwirkten.

Die Lokalisierung und Sichtung der Quellentexte beanspruchte den größten Teil der für die vorliegende Untersuchung zur Verfügung stehenden Zeit. Die in Frage kommenden, häufig nur in geringer Anzahl gedruckten Schriften sind in früherer Zeit kaum systematisch gesammelt worden; hinzu kamen dann die in der Zeit des Dritten Reiches verübten Zerstörungen und Zerstreuungen deutscher und europäischer Judaica-Bestände. Entsprechend aufwendig, nicht selten auch ertraglos, verliefen die Bemühungen um das Material zu dem vorliegenden Beitrag. Hier war die freundliche Hilfe mancher Personen und Institutionen eine große Unterstützung. Wir möchten an dieser Stelle in Dankbarkeit besonders die Kölner Bibliothek Germania Judaica, die Bibliothek des Institutes für die Geschichte der Juden in Deutschland (Universität Hamburg), die Judaica-Abteilung der Stadt- und Universitätsbibliothek in Frankfurt a.M. und die tüchtige Fernleihstelle der Staats- und Universitätsbibliothek Bremen erwähnen, ferner die vertrauensvoll entgegenkommenden und hilfreichen Besitzer der Privatsammlung Hyams (im Marburger Kindheitsmuseum). Wirklich gastfreundlich und engagiert zeigten sich auch Mr. Esra Kahn, Bibliothekar der Jews College Library in London, sowie zu wiederholten Malen die 
Mitarbeiter der Judaica- u. Hebraica-Abteilung in der Königlichen Bibliothek Kopenhagen; in beiden Einrichtungen wurden angenehme Arbeitsmöglichkeiten zur Verfügung gestellt. Schließlich sei auch der Deutschen Forschungsgemeinschaft herzlich gedankt, die unser Forschungsprojekt am Institut für Jugendbuchforschung der Universität Frankfurt a.M. über einen Zeitraum von zwei Jahren mit jeweils einer Halbtagsstelle gefördert hat.

Michael Nagel 


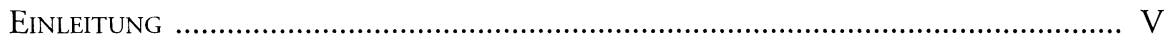

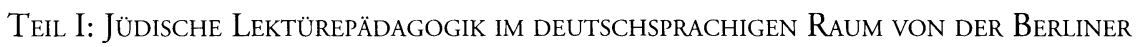
Haskala bis zur Neo-Orthodoxie: Literarische Programme und Kontroversen FÜR DIE JUGEND EINER MinderheIT (Michael Nagel) ................................................ 1

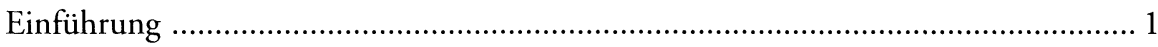

1. Mendelssohns hochdeutsche Pentateuch-Übersetzung:

Der Beginn des Umbruchs im jüdischen Erziehungswesen ................................ 3

2. Lehre und Lektüre nach der traditionellen Auffassung: Hinweise und Gebote

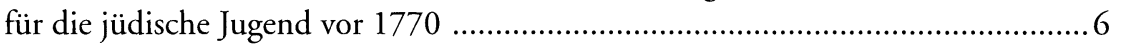

2.3 Lektüre und Bildungsweg der männlichen Jugend ................................. 12

2.4 Nichtjüdische Bücher: Zwischen Vorbehalten und Offenheit .................... 15

3. Die ersten jüdischen Schulbücher: Innerjüdische und überkonfessionelle Diskussion 19

3.1 Lesebuch für Jüdische Kinder. Zum Besten der jüdischen Freyschule, Berlin 1779

3.2 Lesebuch für die jüdische Jugend der deutschen Schulen im Königreiche Böhmen. Bestehend in der Anleitung zur Rechtschaffenheit, Prag: Kaiserl. königl.Normalschule 1781

3.2.1 Der böhmische Schulreformer Ferdinand Kindermann v. Schulstein

3.2.2 Das Verfahren der Adaption von nichtjüdischen Lehrbüchern

3.2.3 Eine nichtjüdische Initiative zur Umgestaltung des jüdischen Erziehungswesens und Mendelssohns Bedenken dazu

3.2.4 Die »Rohigkeit der jüdischen Jugend «: Sittlich-moralische Bildung durch die Exempel-Erzählung

3.2.5 Exkurs: Ein jüdische Lesebuch auch für die Tolerierten Juden in Wien?

3.2.6 Das Lesebuch von 1781 und die neue Normalschule im Urteil des zeitgenössischen Prager Judentums 
3.2.7 Das jüdische Lesebuch und die jüdische Normalschule in der öffentlichen Aufmerksamkeit

3.3 Nathan, Wolf [ben] Abraham: Jüdische Religionsstütze,

oder Grundsätze der Jüdischen Religion aus den heiligen

Büchern, Talmud und den vorzüglichsten Rabbinen

zusammengetragen, Dessau 1782.

4. Die Jüdische Tradition und das Bildungskonzept der Spätaufklärung

5. Zur jüdischen Lektürepädagogik zwischen der Epoche Mendelssohns und dem Erziehungskonzept Samson Raphael Hirschs: Ein Ausblick.

TeIl II: Von Der Neo-Orthodoxie bis ZUM Dritten Reich

(Gabriele v. Glasenapp)

1. Neo-Orthodoxe Konzepte der Kinder- und Jugendliteratur 79

2. Die jüdische Rezeption der Jugendschriftenbewegung 94

3. Literarische Erziehung in der Frühzeit des deutschen Zionismus

4. Neue literaturpädagogische Ansätze nach dem Ende des Ersten Weltkrieges ..... 127

5. Zwischen Unterdrückung, Selbstbehauptung und Emigration: Literarische Erziehung unter nationalsozialistischer Herrschaft in den Jahren 1933 bis 1938

ANMERKUNGEN

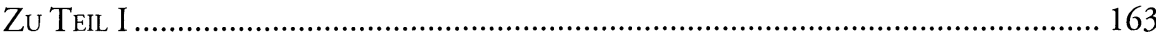

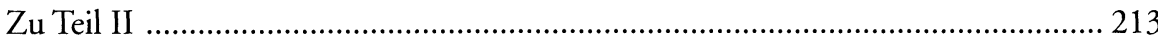

LITERATURVERZEICHNIS

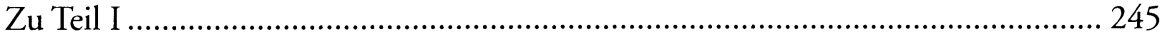

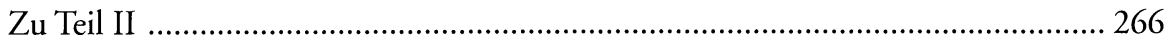

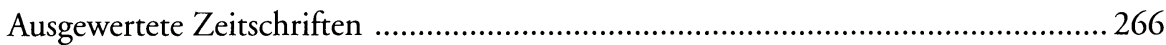

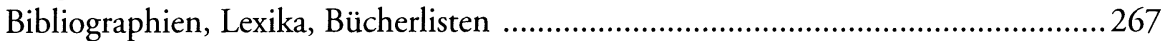

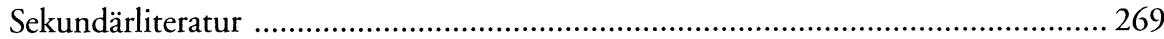

Personenregister

zu Teil I und II 